\title{
CONVERGENCE AND SUBSEQUENTIAL CONVERGENCE OF REGULARLY GENERATED SEQUENCES
}

\author{
SEFA ANIL SEZER AND İBRAHIM ÇANAK
}

Received 03 November, 2013

\begin{abstract}
In this paper we recover convergence and subsequential convergence of a sequence of real numbers regularly generated by another sequence in some sequence spaces under certain conditions. We also give some information about the behavior of a sequence whose generator is given in terms of a moderately divergent sequence.
\end{abstract}

2010 Mathematics Subject Classification: 40A05; 40E05

Keywords: regularly generated sequences, slow oscillation, moderate oscillation, moderate divergence, subsequential convergence, weighted means

\section{INTRODUCTION}

Throughout this paper, $\mathbb{N}_{0}$ will denote the set of all nonnegative integers. Let $u=\left(u_{n}\right)$ be a sequence of real numbers and any term with a negative index be zero. Let $p=\left(p_{n}\right)$ be a sequence of nonnegative numbers such that $p_{0}>0$ and

$$
P_{n}:=\sum_{k=0}^{n} p_{k} \rightarrow \infty, n \rightarrow \infty .
$$

The $n^{\text {th }}$ weighted mean of the sequence $\left(u_{n}\right)$ is defined by

$$
\sigma_{n, p}(u):=\frac{1}{P_{n}} \sum_{k=0}^{n} p_{k} u_{k}
$$

for all $n \in \mathbb{N}_{0}$.

The sequence $\left(u_{n}\right)$ is said to be summable by the weighted mean method determined by the sequence $p$; in short, $(\bar{N}, p)$ summable to a finite number $s$ if

$$
\lim _{n \rightarrow \infty} \sigma_{n, p}(u)=s \text {. }
$$

The difference between $u_{n}$ and its $n^{\text {th }}$ weighted mean $\sigma_{n, p}(u)$, which is called the weighted Kronecker identity, is given by

$$
u_{n}-\sigma_{n, p}(u)=V_{n, p}(\Delta u),
$$


where

$$
V_{n, p}(\Delta u):=\frac{1}{P_{n}} \sum_{k=0}^{n} P_{k-1} \Delta u_{k}
$$

and

$$
\Delta u_{n}=u_{n}-u_{n-1} .
$$

The $(\bar{N}, p)$ summability method is regular if and only if $P_{n} \rightarrow \infty$ as $n \rightarrow \infty$. If $p_{n}=$ 1 for all $n \in \mathbb{N}_{0}$, then $(\bar{N}, p)$ summability method reduces to Cesàro summability method.

A sequence $\left(u_{n}\right)$ is slowly oscillating [14] if

$$
\lim _{\lambda \rightarrow 1^{+}} \limsup _{n \rightarrow \infty} \max _{n+1 \leq k \leq[\lambda n]}\left|u_{k}-u_{n}\right|=0,
$$

where $[\lambda n]$ denotes the integer part of $\lambda n$.

The space of all slowly oscillating sequences is denoted by $\mathcal{8 \mathcal { O }}$. Dik [9] proved that if a sequence $\left(u_{n}\right)$ is slowly oscillating, then $\left(V_{n, 1}(\Delta u)\right)$ is bounded and slowly oscillating.

A generalization of slow oscillation is given as follows.

A sequence $\left(u_{n}\right)$ is moderately oscillating [14] if

$$
\limsup _{n \rightarrow \infty} \max _{n+1 \leq k \leq[\lambda n]}\left|u_{k}-u_{n}\right|<\infty
$$

for $\lambda>1$. The space of all moderately oscillating sequences is denoted by $\mathcal{M O}$.

Set

$$
t_{n}=n \frac{p_{n}}{P_{n-1}},
$$

for $n \in \mathbb{N}_{0}$. We say that $\left(u_{n}\right)$ is regularly generated by a sequence $\alpha=\left(\alpha_{n}\right)$ in some sequence space $\mathcal{A}$ and $\alpha$ is called a generator of $\left(u_{n}\right)$ if

$$
u_{n}=\alpha_{n}+\sum_{k=1}^{n} \frac{t_{k}}{k} \alpha_{k} .
$$

The space of all sequences which are regularly generated by sequences in $\mathcal{A}$ is denoted by $U(\mathcal{A})$.

If $\left(u_{n}\right)$ is regularly generated by a sequence $\left(\alpha_{n}\right)$ where $\left(\Delta \alpha_{n}\right) \in \mathcal{S O}$, we write $\left(u_{n}\right) \in U\left(\mathcal{S O}_{\Delta}\right)$. If $\left(u_{n}\right)$ is regularly generated by a sequence $\left(\alpha_{n}\right)$ where $\left(\alpha_{n}\right) \in \mathcal{S O}$, we write $\left(u_{n}\right) \in U(\mathcal{O O})$.

A positive sequence $\left(u_{n}\right)$ is O-regularly varying [12] if

$$
\limsup _{n \rightarrow \infty} \frac{u_{[\lambda n]}}{u_{n}}<\infty
$$

for $\lambda>1$ and it is slowly varying if

$$
\lim _{n \rightarrow \infty} \frac{u_{[\lambda n]}}{u_{n}}=1
$$


It was proved by [11] that if a positive sequence $\left(u_{n}\right)$ is O-regularly varying, then $\left(\log u_{n}\right)$ is slowly varying.

A positive sequence $\left(u_{n}\right)$ is moderately divergent [13] if for every $\lambda>1$

$$
u_{n}=o\left(n^{\lambda-1}\right), n \rightarrow \infty
$$

and

$$
\sum_{n=1}^{\infty} \frac{u_{n}}{n^{\lambda}}<\infty
$$

We denote the space of all moderately divergent sequences by $\mathcal{M} D$. Note that every slowly oscillating sequence of positive numbers is moderately divergent.

The convergence of a sequence $\left(u_{n}\right)$ implies that $\left(u_{n}\right)$ is bounded and $\Delta u_{n}=$ $o(1)$ as $n \rightarrow \infty$. But it is clear that the converse of this implication is not true in general. In the case where $\left(u_{n}\right)$ is bounded with $\Delta u_{n}=o(1)$ as $n \rightarrow \infty$, we may not recover convergence of $\left(u_{n}\right)$ but we may have convergence of some subsequences of $\left(u_{n}\right)$. A new kind of convergence is defined as follows (See [8] for more details on subsequentially convergent sequences):

A sequence $u=\left(u_{n}\right)$ is said to be subsequentially convergent if there exists a finite interval $I(u)$ such that all accumulation points of $\left(u_{n}\right)$ are in $I(u)$ and every point of $I(u)$ is an accumulation point of $\left(u_{n}\right)$.

Recently, several results in terms of regularly generated sequences for different purposes have been obtained by Dik et al. [10], Çanak et al. [1], Çanak and Totur [3], Çanak et al. [2], Çanak et al. [7], Çanak and Totur [5] and many more. In this paper, we first recover convergence and subsequential convergence of a sequence which is regularly generated by another sequence in some sequence spaces under certain conditions. Secondly, we give some information about the behavior of a sequence whose generator is given in terms of a moderately divergent sequence.

\section{THE PRELIMINARY RESULTS}

We need the following lemmas for the proof of our results.

Lemma 1 ([8]). Let $\left(u_{n}\right)$ be a bounded sequence of real numbers. If $\Delta u_{n}=o(1)$ as $n \rightarrow \infty$, then $\left(u_{n}\right)$ converges subsequentially.

Lemma 2. If $\left(\sum_{k=1}^{n} t_{k} \alpha_{k}\right)$ is moderately oscillating, then $\left(\sum_{k=1}^{n} \frac{t_{k}}{k} \alpha_{k}\right)$ converges.

Proof. Set $R_{n}:=\exp \left(\left|\sum_{k=1}^{n} t_{k} \alpha_{k}\right|\right)$. Then we have

$$
\frac{R_{[\lambda n]}}{R_{n}} \leq \exp \left(\left|\sum_{k=n+1}^{[\lambda n]} t_{k} \alpha_{k}\right|\right) .
$$


Taking limsup of both sides of (2.1) as $n \rightarrow \infty$ gives

$$
\limsup _{n \rightarrow \infty} \frac{R_{[\lambda n]}}{R_{n}} \leq \exp \left(\limsup _{n \rightarrow \infty}\left|\sum_{k=n+1}^{[\lambda n]} t_{k} \alpha_{k}\right|\right) .
$$

Since $\left(\sum_{k=1}^{n} t_{k} \alpha_{k}\right)$ is moderately oscillating, we have

$$
\limsup _{n \rightarrow \infty} \frac{R_{[\lambda n]}}{R_{n}}
$$

is finite for $\lambda>1$. This says that $\left(R_{n}\right)$ is O-regularly varying. Since $\left(R_{n}\right)$ is Oregularly varying, $\left(\log R_{n}\right)$ is slowly varying. It follows that

$$
\sum_{n=1}^{\infty} \frac{1}{n^{p}}\left|\sum_{k=1}^{n} t_{k} \alpha_{k}\right|^{p}<\infty
$$

for $p>1$. This implies that

$$
\sum_{n=1}^{\infty} \frac{t_{n}}{n} \alpha_{n}<\infty
$$

Lemma 3. If $\left(\sum_{k=1}^{n} \frac{t_{k}}{k} \alpha_{k}\right)$ converges, then $\sigma_{n, p}(\alpha)=o(1), n \rightarrow \infty$.

Proof. Set $\gamma_{n}:=\sum_{k=1}^{n} \frac{t_{k}}{k} \alpha_{k}$. Then we obtain

$$
\alpha_{n}=\frac{P_{n-1}}{p_{n}} \Delta \gamma_{n}
$$

and

$$
\sigma_{n, p}(\alpha)=V_{n, p}(\Delta \gamma)
$$

for $n \in \mathbb{N}_{0}$. Since $\left(\gamma_{n}\right)$ converges, it follows by the weighted Kronecker identity

$$
\gamma_{n}-\sigma_{n, p}(\gamma)=V_{n, p}(\Delta \gamma)
$$

that

$$
V_{n, p}(\Delta \gamma)=o(1), n \rightarrow \infty .
$$

This completes the proof.

Lemma 4 ([6]). Let $\left(p_{n}\right)$ satisfy the condition

$$
1 \leq \frac{P_{n}}{n} \rightarrow 1, n \rightarrow \infty .
$$

If $\left(u_{n}\right)$ is slowly oscillating, then $\left(V_{n, p}(\Delta u)\right)$ is slowly oscillating and bounded.

Lemma 5 ([15]). Let $\left(u_{n}\right)$ be Cesàro summable to s. If $\left(u_{n}\right)$ is slowly oscillating, then $\left(u_{n}\right)$ converges to $s$. 


\section{THE MAIN RESULTS}

Theorem 1. Suppose that

$$
\begin{gathered}
\left(\sum_{k=1}^{n} t_{k} \alpha_{k}\right) \in \mathcal{M} \mathcal{O}, \\
1 \leq \frac{P_{n}}{n} \rightarrow 1, n \rightarrow \infty, \\
t_{n}=O(1), n \rightarrow \infty .
\end{gathered}
$$

If $\left(u_{n}\right) \in U\left(8 \mathcal{O}_{\Delta}\right)$, then $\left(u_{n}\right)$ converges subsequentially.

Proof. Since $\left(u_{n}\right) \in U\left(\mathcal{S O}_{\Delta}\right),\left(u_{n}\right)$ can be written as

$$
u_{n}=\alpha_{n}+\sum_{k=1}^{n} \frac{t_{k}}{k} \alpha_{k},
$$

where $\left(\Delta \alpha_{n}\right) \in \mathcal{S O}$. Moderate oscillation of $\left(\sum_{k=1}^{n} t_{k} \alpha_{k}\right)$ implies convergence of $\left(\gamma_{n}\right)=\left(\sum_{k=1}^{n} \frac{t_{k}}{k} \alpha_{k}\right)$ by Lemma 2 and $\sigma_{n, p}(\alpha)=o(1)$ as $n \rightarrow \infty$ by Lemma 3 . Hence, $\left(u_{n}\right)$ is $(\bar{N}, p)$ summable to the limit of $\left(\gamma_{n}\right)$. By the condition (3.3), it follows that

$$
\frac{\alpha_{n}}{n} \rightarrow 0, n \rightarrow \infty
$$

by Lemma 3. Since $\left(\Delta \alpha_{n}\right) \in \mathcal{S O}$, we have that

$$
\Delta \alpha_{n} \rightarrow 0, n \rightarrow \infty
$$

by Lemma 5 . Taking the backward difference of (3.4), we have

$$
\Delta u_{n}=\Delta \alpha_{n}+\alpha_{n} \frac{p_{n}}{P_{n-1}}
$$

for $n \in \mathbb{N}_{0}$.

It follows by (3.3), (3.5) and (3.6) that

$$
\Delta u_{n}=o(1), n \rightarrow \infty \text {. }
$$

To complete the proof, it suffices to prove that $\left(u_{n}\right)$ is bounded. Applying Lemma 4 to $\left(v_{n}\right)=\left(\sum_{k=1}^{n} \alpha_{k} t_{k}\right)$, and taking $\left(v_{n}\right) \in \mathcal{S O}$ into account, we obtain $\left(V_{n, p}(\alpha t)\right)$ is bounded and slowly oscillating, where $\alpha t=\left(\alpha_{n} t_{n}\right)$.

From the weighted Kronecker identity

$$
S_{n}(\alpha)-\sigma_{n, p}(S(\alpha))=V_{n, p}(\alpha)
$$

where $S(\alpha)=\left(S_{n}(\alpha)\right)=\left(\sum_{k=0}^{n} \alpha_{k}\right)$, we have

$$
\alpha_{n}-\frac{p_{n}}{P_{n-1}} V_{n, p}(\alpha)=\Delta V_{n, p}(\alpha) .
$$


Replacing $\alpha_{n}$ by $\alpha_{n} t_{n}$ in (3.10) and then dividing by $t_{n}$, we have

$$
\alpha_{n}=\frac{V_{n, p}(\alpha t)}{n}+\frac{P_{n-1}}{n p_{n}} \Delta V_{n, p}(\alpha t) .
$$

It follows from (3.11) that $\left(\alpha_{n}\right)$ is bounded. Hence, $\left(u_{n}\right)$ is bounded. By Lemma 1, $\left(u_{n}\right)$ is subsequentially convergent.

Theorem 2. Suppose that

$$
\begin{gathered}
\left(\sum_{k=1}^{n} t_{k} \alpha_{k}\right) \in \mathcal{M O}, \\
1 \leq \frac{P_{n}}{n} \rightarrow 1, n \rightarrow \infty, \\
1<\liminf _{n \rightarrow \infty} \frac{P_{[\lambda n]}}{P_{n}}<\limsup _{n \rightarrow \infty} \frac{P_{[\lambda n]}}{P_{n}}<\infty, \text { for } \lambda>1, \\
1<\liminf _{n \rightarrow \infty} \frac{P_{n}}{P_{[\lambda n]}}<\limsup _{n \rightarrow \infty} \frac{P_{n}}{P_{[\lambda n]}}<\infty, \text { for } 0<\lambda<1, \\
t_{n}=O(1), n \rightarrow \infty .
\end{gathered}
$$

If $\left(u_{n}\right) \in U(\mathcal{S O})$, then $\left(u_{n}\right)$ converges.

Proof. Assume that $\left(u_{n}\right) \in U(\mathcal{O O})$. Then, $\left(u_{n}\right)$ can be written as

$$
u_{n}=\alpha_{n}+\sum_{k=1}^{n} \frac{t_{k}}{k} \alpha_{k}
$$

where $\left(\alpha_{n}\right) \in \boldsymbol{S O}$. From (3.17), we have

$$
V_{n, p}(\Delta u)=V_{n, p}(\Delta \alpha)+\sigma_{n, p}(\alpha) .
$$

Moderate oscillation of $\left(\sum_{k=1}^{n} t_{k} \alpha_{k}\right)$ implies convergence of $\left(\gamma_{n}\right)=\left(\sum_{k=1}^{n} \frac{t_{k}}{k} \alpha_{k}\right)$ by Lemma 2 and $\sigma_{n, p}(\alpha)=o(1)$ as $n \rightarrow \infty$ by Lemma 3. Therefore, $\left(u_{n}\right)$ is $(\bar{N}, p)$ summable to the limit of $\left(\gamma_{n}\right)$.

Since $\left(\alpha_{n}\right)$ is slowly oscillating, $\left(V_{n, p}(\Delta \alpha)\right)$ is bounded and slowly oscillating by Lemma 4.

It follows from (3.18) that $\left(V_{n, p}(\Delta u)\right) \in \delta \mathcal{O}$ and bounded. Since $\left(u_{n}\right)$ is $(\bar{N}, p)$ summable, $\left(u_{n}\right)$ converges to $\lim _{n \rightarrow \infty} \sigma_{n, p}(u)$ by Theorem 6 in [4].

Theorem 3. Suppose that $\left(u_{n}\right)$ is regularly generated by $\left(\alpha_{n}\right)$ and

$$
\frac{p_{n}}{P_{n-1}}-\frac{p_{n+1}}{P_{n}}=O\left(\frac{1}{n^{2}}\right), n \rightarrow \infty .
$$

If

$$
\sum_{k=1}^{n} \alpha_{k}=n^{\gamma} m_{n}
$$




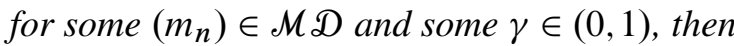

i) $\left(u_{n}\right)$ is $(\bar{N}, p)$ summable.

ii) $u_{n}=\Delta\left(n^{\gamma} m_{n}\right)+\beta_{n}$, where $\beta_{n}=o(1), n \rightarrow \infty$.

iii) $u_{n}=o(n), n \rightarrow \infty$ and $\sum_{n=1}^{\infty} \frac{u_{n}}{n^{2}}<\infty$.

Proof. i) By Abel's partial summation formula, we have

$$
\begin{aligned}
\sum_{k=1}^{n} \frac{p_{k}}{P_{k-1}} \alpha_{k} & =\sum_{k=1}^{n} \frac{p_{k}}{P_{k-1}}\left(S_{k}(\alpha)-S_{k-1}(\alpha)\right) \\
& =\sum_{k=1}^{n} \frac{p_{k}}{P_{k-1}} S_{k}(\alpha)-\sum_{k=1}^{n} \frac{p_{k}}{P_{k-1}} S_{k-1}(\alpha) \\
& =\sum_{k=1}^{n}\left(\frac{p_{k}}{P_{k-1}} S_{k}(\alpha)-\frac{p_{k+1}}{P_{k}} S_{k}(\alpha)\right)+\frac{p_{n}}{P_{n-1}} S_{n}(\alpha)-\frac{p_{1}}{P_{0}} S_{0} \\
& =\frac{p_{n}}{P_{n-1}} S_{n}(\alpha)+\sum_{k=1}^{n-1}\left(\frac{p_{k}}{P_{k-1}}-\frac{p_{k+1}}{P_{k}}\right) S_{k}(\alpha)
\end{aligned}
$$

Since $S_{n}(\alpha)=n^{\gamma} m_{n}$ for some $\left(m_{n}\right) \in \mathcal{M} D$, we have

$$
\frac{p_{n}}{P_{n-1}} S_{n}(\alpha)=O\left(\frac{m_{n}}{n^{1-\gamma}}\right), n \rightarrow \infty .
$$

By moderate divergence of $\left(m_{n}\right)$, we have

$$
\frac{p_{n}}{P_{n-1}} S_{n}(\alpha)=o(1), n \rightarrow \infty .
$$

The second term on the right of (3.21) converges by (3.19). It follows from the representation

$$
u_{n}=\alpha_{n}+\sum_{k=1}^{n} \frac{p_{k}}{P_{k-1}} \alpha_{k},
$$

that $\left(u_{n}\right)$ is $(\bar{N}, p)$ summable.

ii) Note that the sequence $\left(\beta_{n}\right)$ defined by $\beta_{n}=\frac{t_{n} \alpha_{n}}{n}$ for $n \in \mathbb{N}_{0}$ converges to zero. From the representation and the condition (3.20) it follows that

$$
u_{n}=\Delta\left(n^{\gamma} m_{n}\right)+\beta_{n}
$$

where $\beta_{n}=\frac{t_{n}}{n} \alpha_{n}$.

iii) By ii), we have

$$
u_{n}=n^{\gamma} m_{n}-(n-1)^{\gamma} m_{n-1}+\beta_{n} .
$$


Dividing (3.26) by $n$, we have

$$
\frac{u_{n}}{n}=\frac{m_{n}}{n^{1-\gamma}}-\frac{m_{n-1}}{(n-1)^{1-\gamma}}+\frac{\beta_{n}}{n} .
$$

Since $\left(m_{n}\right) \in \mathcal{M} \mathfrak{D}$ and $\beta_{n}=o(1)$, we have

$$
\frac{u_{n}}{n}=o(1), n \rightarrow \infty
$$

By (3.26), we obtain

$$
\sum_{k=2}^{n} \frac{u_{k}}{k^{2}}=\sum_{k=2}^{n} \frac{m_{k}}{k^{2-\gamma}}-\sum_{k=2}^{n} \frac{m_{k-1}}{(k-1)^{2-\gamma}}+\sum_{k=2}^{n} \frac{\beta_{k}}{k^{2}} .
$$

Taking the limit of both sides of (3.29) as $n \rightarrow \infty$, we obtain $\sum_{n=1}^{\infty} \frac{u_{n}}{n^{2}}<\infty$.

\section{REFERENCES}

[1] İ. Çanak, M. Dik, and F. Dik, “Conditions for convergence and subsequential convergence,” Appl. Math. Lett., vol. 19, no. 10, pp. 1042-1045, 2006, doi: 10.1016/j.aml.2005.11.015.

[2] İ. Çanak, F. Hasekiler, and D. Kebapc1, "Some Tauberian theorems for regularly generated sequences," Comput. Math. Appl., vol. 62, no. 21, pp. 4486-4491, 2011, doi: 10.1016/j.camwa.2011.10.027.

[3] İ. Çanak and Ü. Totur, "A note on Tauberian theorems for regularly generated sequences," Tamkang J. Math., vol. 39, no. 2, pp. 187-191, 2008, doi: 10.5556/j.tkjm.39.2008.29.

[4] İ. Çanak and Ü. Totur, "Some Tauberian theorems for the weighted mean methods of summability," Comput. Math. Appl., vol. 62, pp. 2609-2615, 2011, doi: 10.1016/j.camwa.2011.07.066.

[5] İ. Çanak and Ü. Totur, "Some Tauberian conditions for Cesàro summability method," Math. Slovaca, vol. 62, no. 2, pp. 271-280, 2012, doi: 10.2478/s12175-012-0008-y.

[6] İ. Çanak and Ü. Totur, "Tauberian theorems for the $(J, p)$ summability method," Appl. Math. Lett., vol. 25, no. 10, pp. 1430-1434, 2012, doi: 10.1016/j.aml.2011.12.017.

[7] İ. Çanak, Ü. Totur, and M. Dik, "On Tauberian theorems for $(A, k)$ summability method," Math. Slovaca, vol. 61, no. 6, pp. 993-1001, 2011, doi: 10.2478/s12175-011-0064-8.

[8] F. Dik, "Tauberian theorems for convergence and subsequential convergence with moderately oscillatory behavior," Math. Morav., vol. 5, pp. 19-56, 2001.

[9] M. Dik, "Tauberian theorems for sequences with moderately oscillatory control modulo," Math. Morav., vol. 5, pp. 57-94, 2001.

[10] M. Dik, F. Dik, and İ. Çanak, "Classical and neoclassical Tauberian theorems for regularly generated sequences," Far East J. Math. Sci. (FJMS), vol. 13, no. 2, pp. 233-240, 2004.

[11] D. Natsis, Convergence of Fourier series and representations of Fourier and Fourier-Stieltjes coefficients, ser. Ph. D. Dissertation. University of Missouri-Rola, 1991.

[12] Č. V. Stanojević, "O-regularly varying convergence moduli of Fourier and Fourier-Stieltjes series," Math. Ann., vol. 279, no. 1-2, pp. 103-115, 1987, doi: 10.1007/BF01456193.

[13] Č. V. Stanojević, "Fourier and Fourier-Stieltjes series with compacticity property." Moscow: Steklov Mathematical Institute, 1990.

[14] Č. V. Stanojević, Analysis of Divergence: Control and Management of Divergent Process, ser. Graduate Research Seminar Lecture Notes, 1998.

[15] Č. V. Stanojević, Analysis of Divergence: Applications to the Tauberian theory, ser. Graduate Research Seminars, 1999. 
Authors' addresses

\section{Sefa Anıl Sezer}

Istanbul Medeniyet University, Department of Mathematics, 34720 Istanbul, Turkey Current address: Ege University, Department of Mathematics, 35100 Izmir, Turkey

E-mail address: sefaanil.sezer@medeniyet.edu.tr

\section{İbrahim Çanak}

Ege University, Department of Mathematics, 35100 Izmir, Turkey

E-mail address: ibrahimcanak@yahoo.com 